\title{
Controlled Electricity Distribution Network Black Start with Energy Storage System Support
}

\author{
Alessandro Di Giorgio, Alessandro Giuseppi, Francesco Liberati, Antonio Pietrabissa
}

\begin{abstract}
This paper presents a simple optimal procedure for controlled electricity start for electricity distribution grids in reaction to adverse events or malicious cyber attacks resulting in the interruption of the main power supply from transmission network. The islanded operation is supposed to be guaranteed by an electric energy storage system, which covers the network imbalance between demand and supply from distributed generation during the sequential reconnection of medium voltage feeders. The objective is to restore the service to the consumers while avoiding violation of technical constraints in terms of storage power flow and battery capacity. The discussion of simulation results assesses the effectiveness of the proposed approach in the context of a simplified network model and gives rise to relevant remarks and requirements for further developments.
\end{abstract}

\section{INTRODUCTION}

The increasing presence of distributed renewable generation opens new possibilities for faults and cyber attacks mitigation in the distribution network. One example is the socalled islanded operation of the distribution network, studied in reference [1]. At distribution level, the problem of black start traditionally consists in finding an optimal reconnection order of the various secondary substations, exploiting selfstarting controlled power plants, such as hydroelectric units, diesel generators and gas turbines after a power outage [2], during which the connection between the HV and MV grids is interrupted. The procedure aims at restoring the generation from bigger power plants by feeding a portion of the network with the self-starting units. The presence of an Energy Storage System (ESS) can support the islanded operation of the networks, as it can either absorb or provide the balancing power that normally would flow in the primary substation, allowing the exploitation of uncontrollable distributed generation, such as photovoltaic and other renewable plants. The ESS also provides the reference values for voltage and frequency. In recent years several possible applications of ESSs have been investigated at transmission [3]-[5], distribution [6]-[10], microgrid [11], [12] and consumer [13] level. It is then of interest to study another possible application of this technology, whose distribution is expected to grow over the following years, due to its great applications in the field of renewable resources [14]. This work has

A. Di Giorgio, A. Giuseppi, A. Pietrabissa are with the Department of Computer, Control and Management Engineering Antonio Ruberti, at Sapienza University of Rome, Via Ariosto 25, 00185, Rome, Italy, e-mail: \{digiorgio,giuseppi,pietrabissa\}@diag.uniroma1.it.

F. Liberati is with the SMART Engineering Solutions \& Technologies (SMARTEST) Research Center, eCampus University, Via Isimbardi 10 22060 Novedrate (CO), Italy, e-mail: \{francesco.liberati\}@uniecampus.it.

This work has been carried out in the framework of the ATENA project [Grant Agreement \#700581] partially funded by the EU. been developed in the context of cyber-physical security for Critical Infrastructures (CI), and proposes a control strategy that enables the power grid to operate even after a distributed power outage in islanded mode, maximizing the system survival time, hence preventing the propagation of damage caused by attacks or faults to other CIs interdependent with the electricity grid, such as the gas pipeline and the water system (see, e.g., the EU projects MICIE and ATENA [15][17])

Section II discusses the reference scenario and present the problem under investigation. Section III details the mathematical formulation of the proposed approach for controlled black start. Section III-D discusses the results of the work and a set of additional requirement to be taken into account for future developments. Finally the conclusions are drawn in Section IV.

\section{PROBLEM DESCRIPTION}

This section rolls out the main aspects of distribution network black start in presence of ESS. The simplified but still significant reference scenario is depicted in Fig. 1, where a distribution system departing from two HV/MV substations is considered. Each substation is equipped with an ESS, which is connected to the MV busbar through a circuit breaker; also a set of MV feeders departs from the busbar and is supposed to end at the MV busbar of the other substation. The feeders are equipped with remotely controlled circuit breakers at their top and distributed switches, by using which it is possible to decide disconnecting (reconnecting) portions of the grid and their assignment to substations; as customary in distribution network operation, despite the electrical connection between substations through a distribution path is possible, the network will be considered radially operated. Loads and distributed generators are connected alongside the feeders, so that different portions of the network can behave either as sinks or sources of active power.

During normal operation, for a given network configuration, the main power supply, together with frequency and voltage regulation services are provided by the transmission network through the HV/MV substations; in this situation the circuit breakers and switches are typically operated for isolation of faults occurring in the distribution grid and the consequent restoration of the power supply service. Here a different situation is considered, in which the power flow in the substations is interrupted, due to a fault in the transmission network or a malicious cyber attack to the distribution system operator's SCADA system. In such a situation the absence of voltage feeding is detected by the 


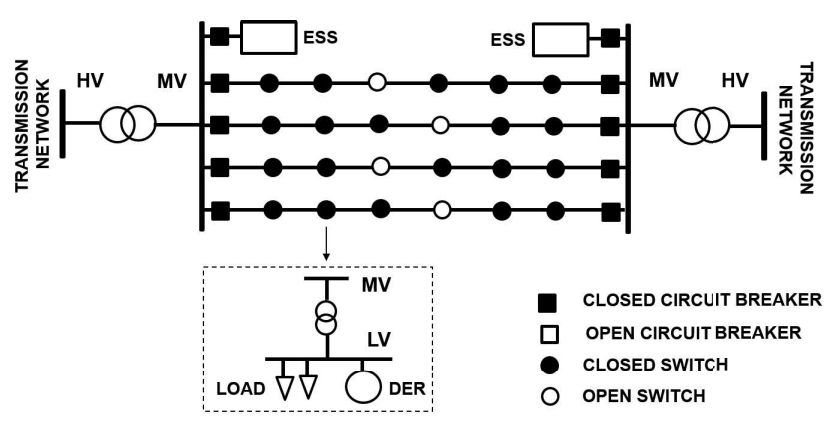

Fig. 1. Reference scenario.

network protection systems, which results in all the circuit beakers and switches to open up, also including the ones disconnecting the distributed generation; detection of such a condition prevents the typical distribution system operational strategy to take place, with open breakers and switches constituting the initial condition from which the black start procedure is initialized. For ease of discussion, at first it will be assumed that the configuration of trees in the network is given a priori, meaning that the black start problem is logically restricted to the one of reconnecting MV portions of feeders within each independent tree, then restoring the power supply service making use of the associated ESS.

Within each tree, the procedure starts with the reclosure of the ESScircuit breaker, by which the voltage on the MV busbar of the substation is restored. Following that proper actions on the feedercircuit breakers and switches have to be taken. Due to the limited ESS capacity in terms of charging/discharging active power and battery, it is typically not possible to reconnect all the loads and distributed generators at the same time. Indeed it is straightforward to realize that whatever set of reconnected loads and generators characterized by a net active power exceeding the nominal ESS power rate would imply the ESS protection to command the disconnection of the device from the grid, and then a new blackout; at the same time, highly imbalanced configurations reduce the grid survivability due to the fast saturation of ESSstate of charge to the upper or lower bound. Then in this simplified scenario the black start problem in presence of ESS can be seen as the one of computing the optimal sequence of breakers and switches reclosures that allows to reconnect the largest possible portion of the distribution grid while guaranteeing feasible ESS operation over time. As described in the following, a natural formulation of this simplified problem raises a number of remarks and requirements to be taken into account for a complete formulation of the black start procedure, in which the proper configuration of distribution trees in combination with an optimal reconnection strategy allows to improve efficiency in terms of network survivability and size of reconnected grid.

\section{PROBLEM FORMALIZATION}

\section{A. Assumptions}

The black start problem described in the following is based on a set of assumptions allowing the modeling of the problem at a simplified level which still captures the fundamental aspects described in the previous section. These are listed in the following.

- Each LV/MV substation alongside the MV feeders is associated with a remotely controlled switch installed at the top of the line departing from it towards downstream sections; reclosure of a switch implies the restoration of voltage feeding on the downstream section of the feeder and reconnection of loads and distributed generators connected to the next MV/LV substation.

- The network is considered to be lossless. This implies the possibility of associating a net aggregated active power value a priori to each reconnected portion of the grid, without taking into account the dependence of line losses on the current provided to downstream substations. The equation for active power flow in the network are consequently neglected.

- Transient effects after reconnection of loads and distributed generators at the same substation are neglected, implying the net active power to istantaneously set to the $a$ priori value. In order to minimize the effect of this assumption, only one switch is actuated at each sampling time. Also, in order make this assumption reasonable, the time between two consecutive reconnections is chosen larger not only than the typical electrical time constants (in the range of tens of milliseconds), but especially than the time of voltage feeding experienced by distributed generators protection systems before executing the reconnection (in the range of seconds).

- The reclosure of a switch implies the impossibility of re-opening it, since it can be actuated only in absence of current flowing towards downstream sections. This class of switches represents the majority of disconnectors commonly used in most distribution networks.

- Reactive power flows and voltage magnitudes along the feeders are not considered in the problem formulation, assuming their possible excursion validated at the moment of configuring the tree departing from the HV/MV substation.

\section{B. Optimal Black Start Procedure}

This subsection presents the optimization problem at the basis of the proposed optimal black start procedure for a given tree of the distribution network, as resulting from the analysis of the previously introduced general problem description and assumptions. In what follows ESS variables and parameters will be denoted as follows: $x(k)$ represents the ESS state of charge at time $k, x^{\min }$ and $x^{\max }$ its minimum and maximum values respectively, $P^{s, n o m}$ the nominal charging and discharging power. On network side $I$ represents the set of feeders departing from the HV/MV substation, $J_{i}$ denotes the set of breakers and switches along 
the $i$-th feeder, $u_{i} j(k)$ is a binary variable equal to 1 in case the switch $j \in J_{I}$ is closed, to 0 otherwise; $P_{i j}$ denotes the net aggregated active power associated to the MV/LV substation which is reconnected when $u_{i j}=1$. Finally $T$ and $K$ denotes the sampling time and the set of steps needed to execute the procedure respectively.

The dynamics affecting the feasibility of the black start procedure is the one of the ESS state of charge, which is modeled as an integrator as follows:

$$
x(k+1)=x(k)-T \sum_{i \in I} \sum_{j \in J_{i}} P_{i j} u_{i j}(k)
$$

Notice that the power to be sustained by the ESS at time $k$ depends on the portion of the grid actually reconnected at that time. This charging/dischaging power is subject to upper and lower bounds as follows:

$$
-P^{s, n o m} \leq \sum_{i \in I} \sum_{j \in J_{i}} P_{i j} u_{i j}(k) \leq P^{s, n o m} \quad \forall k \in K
$$

Also the state of charge is subject to upper and lower bounds at each time of the black start procedure, as follows:

$$
-x^{\min } \leq x(k) \leq x^{\max } \quad \forall k \in K
$$

Then it is worth noting that feasibility of ESS operation depends on the actual grid loading over the time, which is a function of the reconfiguration variables to be determined by solving the proposed optimization problem.

In order to reconnect the customers as fast as possible, the following constraint is introduced, which implies the reclosures to start from the top of each feeder and then an increase of reconnected customers at each step.

$$
u_{i j}(k) \leq u_{i, j-1}(k) \quad \forall i \in I, j \in J_{i}, k \in K
$$

Due to the technological limitation of switches commonly used in distribution network operation, a constraint is introduced to avoid the opening of on-load switches as follows:

$$
u_{i j}(k) \leq u_{i j}(k+1) \quad \forall i \in I, j \in J_{i}, k \in K
$$

Finally the following constraint is introduced to force the actuation of no more than one switch at each step of the procedure. An inequality is here considered instead of a strict equality to allow the problem to be feasible even in case reconnection of the whole grid is not possible by means of the ESS only.

$$
\sum_{i \in I} \sum_{j \in J_{i}}\left[u_{i j}(k+1)-u_{i j}(k)\right] \leq 1 \quad \forall k \in K
$$

Several objective functions can be introduced to drive the optimal sequence of reconnections. Possible objectives ranges from the need of keeping the ESS state of charge near to a safe value, allowing a long survivability of reconnected portions of the grid, to restoring the service as fast as possible, to the maximization of the number of reconnected customers. The first cost function that we are going to present takes the following form:

$$
\min \sum_{k \in K}\left(x(k)-x^{r e f}\right)^{2}
$$

This single term is able to model the need to keep the state of charge of the ESS near a reference during the whole black start procedure, chosen in such a way to be able to compensate eventual unpredicted variations in the power that the device needs to provide/absorb in order to maintain the balance. Furthermore, assuring that the state of charge is close to a defined value, it is possible to calculate an expected survival time for the system, given by the ratio between this reference and the aggregated active power connected to the primary substation at the end of the black start procedure. Additional emphasis on the survival time can be given considering also the following term in the cost function:

$$
\min \left(x\left(k^{f i n}\right)-x^{r e f}\right)^{2} \quad k^{f i n} \in K
$$

Where $k^{\text {fin }}$ represents the last time step for the completion of the procedure. Notice that this term alone does not guarantee a safe evolution of the state of charge of the ESS, but instead limits only its final value. Furthermore in the case where at the end of the procedure all substations are reconnected $k^{f i n}$ is equal to their number, but in general, if the whole grid cannot be reconnected with only one ESS , $k^{\text {fin }}$ may be lower. Other objectives of interest may be the number of reconnected customers, or the prioritization of the reconnection of certain substations. Both this aspects can be modeled by a reward function:

$$
\max \sum_{k \in K} W(i, j) u_{i j}(k)
$$

where the weighting term $W(i, j)$ depends both on the number, their requested load, and the prioritization of the consumers connected to substation which is reconnected when $u_{i} j=1$. The terms above can be linearly combined with opportune weights to reflect the desired goal of the procedure. In the following, given its simpler nature, and the fact that is able to capture multiple relevant system properties, the target function considered will be (7).In the light of the above, the black start problem in presence of ESS support can be stated as follows.

Problem 1:ESS based distribution network black start problem. For a given network whose connection with the HV grid has been interrupted, and whose breakers and switches have all be opened, find the optimal reconnection strategy of the secondary substations minimizing (7) by means of an Energy Storage System to provide i) balancing power and ii) references for frequency and voltage, subject to the dynamics (1) with initial condition $x(0)=x^{r e f}$ and constraints (2)(6). Since the $u_{i j}$ 's are Boolean variables, the described optimization problem is a binary linear programming problem, that can be efficiently solved by standard methods.

Remarks. The problem formulated above takes in to account only the case where a single ESS is available. Due to the presence, for standard fault isolation and service restoration 
procedure, of normally open tie switches between feeders connected to different primary substations, it is in principle possible to reconfigure the network, as in Fig. 1, in such a way that the various $E S S$ are exploited optimally. A reconnection strategy optimal for the single ESS problem may lead to a configuration where the power drained from the ESS is close to its nominal value due to the surplus of loads reconnected with respect to distributed generators, imposing a relevant constraint on the system survival time. On the other hand, another neighboring primary substation could have the opposite problem, where its ESS is absorbing a high quantity of power. Ideally, to maximize the operational time of the two portion of the grid, we could imagine to dynamically reconfigure the network, aiming to keep the state of charge of the ESS as close as possible to the desired reference value. In practice, this dynamic reconfiguration would cause several repeated interruption of service to the consumers, causing severe discomforts and impacting negatively the performance indexes of the operator. In order to extend as much as possible the operational time of the network, while affecting the consumers as little as possible, a reconfiguration algorithm that aims to maximize the steady state survival time of the system is proposed (i.e. an algorithm that does not operate the switches after the procedure has ended). The following formulation makes the same assumptions presented in Section III.A, and additionally considers the presence two ESS at primary substations connected by tie switches as in Fig. 2, where a partition of the network in two separated islands, sustained by the two ESS, is depicted.

\section{Network Configuration}

The cost function in this case needs to drive the system as close as possible to a state of equilibrium. This happens when the aggregated active power, attached at the end of the procedure to each ESS, is close to zero. To keep a more general formulation, we can write:

$$
\min \left(P_{E S S_{1}}-P_{E S S_{1}}^{r e f}\right)^{2}+\left(P_{E S S_{2}}-P_{E S S_{2}}^{r e f}\right)^{2}
$$

where $P_{E S S_{1}}^{r e f}$ and $P_{E S S_{2}}^{r e f}$ represent a target reference value for the steady state power output of the ESSsand the aggregated active powers absorbed/provided by the ESSs, respectively, and $P_{E S S_{1}}$ and $P_{E S S_{2}}$ are defined as follows:

$$
\begin{aligned}
& P_{E S S_{1}}=\sum_{i \in I} \sum_{j \in J_{i}^{\prime}} P_{j i} \delta_{j i} \\
& P_{E S S_{2}}=\sum_{i \in I} \sum_{j \in J_{i}^{\prime}} P_{j i} \bar{\delta}_{j i}
\end{aligned}
$$

where $J_{i}^{\prime}$ represents the set of all the breakers and switches connected to the feeder $i$. If we denote with $J^{1}$ and $J^{2}$ the sets of breakers and switches originally connected to the substations 1 and 2, respectively, it follows $J^{\prime}=J^{1}+J^{2}$. The binary variables $\delta_{i j}$ and $\bar{\delta}_{j i}$ satisfy:

$$
\bar{\delta}_{i j}=1-\delta_{i j} \quad \forall(i, j)
$$

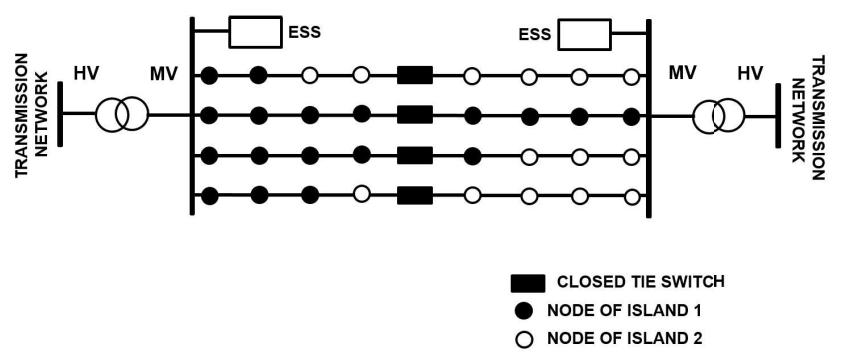

Fig. 2. Reference scenario for the network configuration problem.

$$
\delta_{j, i} \geq \delta_{j, i+1} \quad \forall(i, j)
$$

$\delta_{j i}=1\left(\delta_{j i}=0\right)$ means that the load attached to the switch $\mathrm{i}, \mathrm{j}$ is part of the portion of the grid that will be reconnected to the first (second) ESS.

Equation (13) is the definition of the negated variable, $\bar{\delta}_{j i}$, while (14) is the connectivity and radiality condition for this scenario. In order to minimize the number of nodes that are moved from their original substation to the other, an additional term in the cost function (7) is considered:

$$
\sum_{i \in I}\left(\sum_{j \in J^{1}} \bar{\delta}_{i j}+\sum_{j \in J^{2}} \delta_{i j}\right)
$$

The resulting multi-objective cost function is

$$
\begin{gathered}
\min \left(P_{E S S_{1}}-P_{E S S_{1}}^{r e f}\right)^{2}+\left(P_{E S S_{2}}-P_{E S S_{2}}^{r e f}\right)^{2}+ \\
\alpha \sum_{i \in I}\left(\sum_{j \in J^{1}} \bar{\delta}_{i j}+\sum_{j \in J^{2}} \delta_{i j}\right)
\end{gathered}
$$

where $\alpha$ represents a cost of reconfiguration for each node. The coefficient $\alpha$ has to be scaled accordingly to balance the loads between the two ESS: too big values will completely prevent the algorithm from reconfiguring even a single node from one subnetwork to the other, while values too small could lead to configurations where all loads are attached to a single ESS, leaving the other unused.

This formulation is provided to validate a possible solution to the issues presented in the remarks of Section III.B, in future works it should be generalized to the case with $n$ different ESS, and consider more general radiality constraints as the ones presented in references [18] and [19].

Problem 2: Network reconfiguration for black start in presence of two ESSs. For a given network whose connection with the HV grid has been interrupted, and whose breakers and switches have all be opened, divide the network in two islands, minimizing (16) and respecting constraints (11)-(14).

The black start procedure in presence of two primary substations equipped with ESSs can hence be summarized as follows:

1) Solve problem 2 to find an optimal partition of the network in two islands fed by the ESSs

2) Solve problem 1 for each ESS, obtaining the optimal reconnection strategy of the secondary substations. 


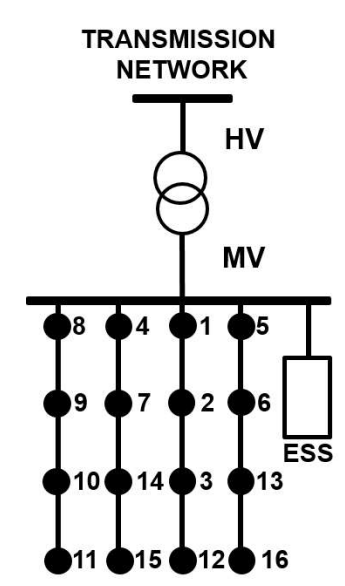

Fig. 3. Network topology for the first simulation with reconnection order.

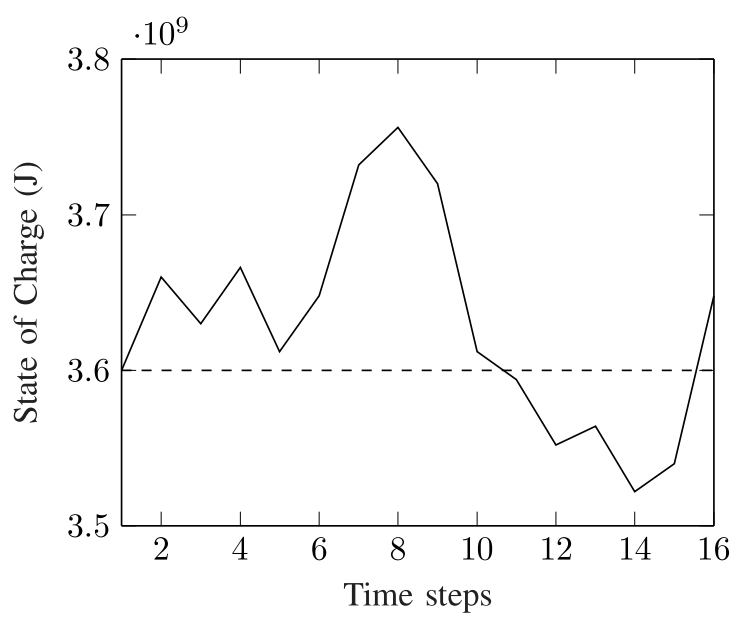

Fig. 4. State of charge in Jouls of the ESS in the first simulation, $x^{r e f}$ dashed.

\section{Simulation Results and Discussion}

Simulation results presented in this paper are aimed at showing the validity of the proposed approach to network reconfiguration to prevent and counteract natural or malicious adverse events. For the first simulation the topology of the network is represented in Fig.3. The aggregated loads $P_{i j}$ are reported in MW in the matrix below, where the element $i, j$ corresponds to $P_{i j}$ and negative values represent power generation.

$$
P=\left(\begin{array}{cccc}
1 & 1.5 & -1 & -1.5 \\
1.2 & 1 & 1.5 & -0.8 \\
-1.5 & -1 & -1.1 & 0.9 \\
0.4 & -1.5 & -0.9 & 1.5
\end{array}\right)
$$

the ESS size considered is $2 \mathrm{MWh}$, with the power output bounded by $P^{s, n o m}=2 \mathrm{MW}$ The time between actions $T$ was set to $60 \mathrm{~s}$, and $x(0)=1 \mathrm{MWh}$ while the evolution of the state of charge of the ESS is reported in Fig. 4. It can be noted that the aggregated active power connected to the primary substation at the end of the black start procedure is

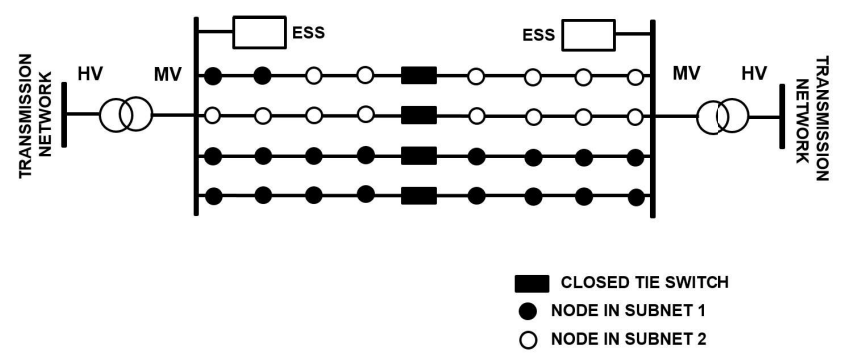

Fig. 5. Reconfiguration of the network in the second simulation.

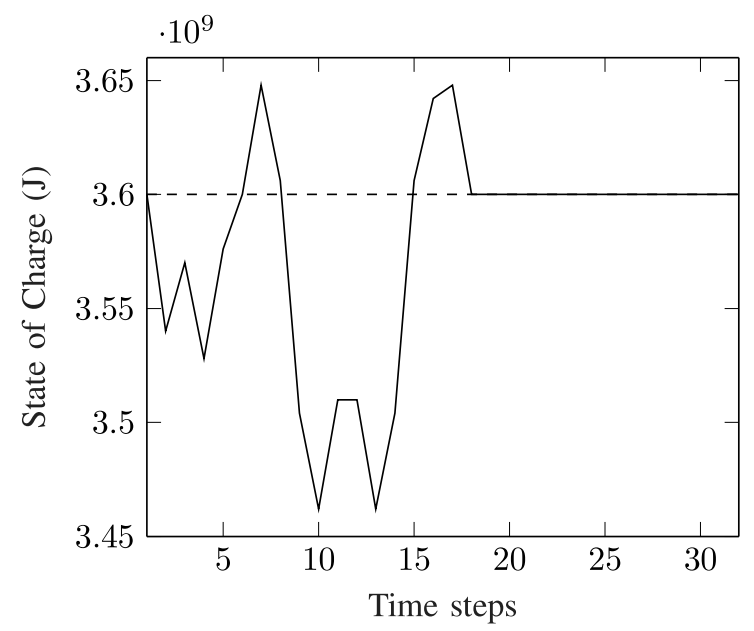

Fig. 6. State of charge in Jouls of the ESS 1 in the second simulation, $x^{\text {ref }}$ dashed.

equal to $-0.3 \mathrm{MW}$, and it is clear from the right part of Fig.4 that the system will drive the ESS to its saturation.

This may not be an issue depending on the forecast restoration time of the connection with the HV network, but in a situation in which the islanded operational time has to be increased (e.g., for more unbalanced networks or long downtime forecast), the solution presented in Section III.C is presented in the following simulation. In this case, the network of the first simulation is connected to another network of the same topology, as in Fig.2, characterized by the matrix load $P^{\prime}$, where the element $i, j$ represents the $\mathrm{j}$ th aggregated load on the $\mathrm{i}$-th feeder, in the network of the second substation:

$$
P^{\prime}=\left(\begin{array}{cccc}
-0.4 & -1 & -1 & 0.6 \\
0.8 & 1.1 & 0.5 & -1 \\
0.8 & 0.5 & -0.5 & 0.6 \\
-1.5 & 0.9 & -0.5 & 0.4
\end{array}\right)
$$

In this network the aggregated active power is $+0.3 \mathrm{MW}$, it is then possible to reconfigure the two networks in order to balance the power consumption in steady state. Simulating the scenario presented, with $\alpha=0.0001$ the networks is reconfigured as shown in Fig.5, archiving perfect balance in steady state for both subnetworks.

The state of charge for the first ESS is reported in Fig.6. It's evident how in this case the storage will be able to 
sustain the network for a longer time. In the two simulations presented, the power imbalance was limited, but as we can see from Figs. 4 and 6 the evolution of the state of charge of the ESSs is bounded by $\sim 5 \%$ variations, providing hence a wide control margin for more unbalanced networks. The limitation on the maximum power output of the ESS is more severe, since heavy uncontrollable loads/generators may not be physically reconnectable to the ESS. To overcome this issue techniques such as load shedding can be used, or it could be reasonable to aim to restore the power only to a set of the substations. Another possible approach that will be further investigated in future works is the reconfiguration of the part of the network that is yet to be reconnected, balancing in advance its aggregated power demand in view of the future reconnection.

\section{CONCLUSIONS}

The paper introduced a first analysis to the black start problem, in presence of distributed energy generation and Energy Storage Systems (ESSs), highlighting the crucial aspects that need to be addressed, and the physical and technological limitations that are to be taken in to account. Future works should focus on the generalization of the proposed formulation, relaxing the assumptions made, for example by introducing power flow equations and considering the presence of multiple ESSs distributed on any substation of the grid. The simplified, but still relevant, formulation introduced has been validated by proof of concept simulations, that highlight its limitations but also enable a relevant analysis of the problem.

\section{ACKNOWLEDGMENT}

The authors gratefully acknowledge the CRAT team involved in the ATENA project and the other ATENA project members for the fruitful discussions over the paper's matters.

\section{REFERENCES}

[1] J. P. Lopes, C. Moreira, F. Resende, et al., "Microgrids black start and islanded operation," in 15th Power systems computation conference (PSCC), Liege, 2005.

[2] J. W. Feltes and C. Grande-Moran, "Black start studies for system restoration," in 2008 IEEE Power and Energy Society General Meeting - Conversion and Delivery of Electrical Energy in the 21st Century, July 2008, pp. 1-8.

[3] A. Di Giorgio, F. Liberati, and A. Lanna, "Real time optimal power flow integrating large scale storage devices and wind generation," in Control and Automation (MED), 2015 23th Mediterranean Conference on. IEEE, 2015, pp. 480-486.

[4] K. M. Chandy, S. H. Low, U. Topcu, and H. Xu, "A simple optimal power flow model with energy storage," in Decision and Control (CDC), 2010 49th IEEE Conference on. IEEE, 2010, pp. 1051-1057.

[5] D. Gayme and U. Topcu, "Optimal power flow with large-scale storage integration," IEEE Transactions on Power Systems, vol. 28, no. 2, pp. 709-717, 2013

[6] A. Di Giorgio, F. Liberati, and A. Lanna, "Electric energy storage systems integration in distribution grids," in Environment and Electrical Engineering (EEEIC), 2015 IEEE 15th International Conference on. IEEE, 2015, pp. 1279-1284.

[7] A. Di Giorgio, F. Liberati, A. Lanna, A. Pietrabissa, and F. D. Priscoli, "Model predictive control of energy storage systems for power tracking and shaving in distribution grids," IEEE Transactions on Sustainable Energy, 2016
[8] A. Nagarajan and R. Ayyanar, "Design and strategy for the deployment of energy storage systems in a distribution feeder with penetration of renewable resources," IEEE Transactions on Sustainable Energy, vol. 6, no. 3, pp. 1085-1092, 2015.

[9] X. Ke, N. Lu, and C. Jin, "Control and size energy storage systems for managing energy imbalance of variable generation resources," IEEE Transactions on Sustainable Energy, vol. 6, no. 1, pp. 70-78, 2015.

[10] S. Manfredi, M. Pagano, and R. Raimo, "Ultracapacitor-based distributed energy resources to support time-varying smart-grid power flows," in Power Electronics, Electrical Drives, Automation and Motion (SPEEDAM), 2012 International Symposium on. IEEE, 2012, pp. 1148-1153.

[11] Y. Levron, J. M. Guerrero, and Y. Beck, "Optimal power flow in microgrids with energy storage," IEEE Transactions on Power Systems, vol. 28, no. 3, pp. 3226-3234, 2013.

[12] A. Di Giorgio, F. Liberati, R. Germana, M. Presciuttini, L. R. Celsi, and F. D. Priscoli, "On the control of energy storage systems for electric vehicles fast charging in service areas," in Control and Automation (MED), 2016 24th Mediterranean Conference on. IEEE, 2016, pp. 955-960.

[13] A. Di Giorgio, L. Pimpinella, and F. Liberati, "A model predictive control approach to the load shifting problem in a household equipped with an energy storage unit," in Control \& Automation (MED), 2012 20th Mediterranean Conference on. IEEE, 2012, pp. 1491-1498.

[14] H. Ibrahim, A. Ilinca, and J. Perron, "Energy storage systemscharacteristics and comparisons," Renewable and Sustainable Energy Reviews, vol. 12, no. 5, pp. 1221 - 1250, 2008. [Online]. Available: http://www.sciencedirect.com/science/article/pii/S1364032107000238

[15] F. Caldeira, M. Castrucci, M. Aubigny, D. Macone, E. Monteiro, F. Rente, P. Simões, and V. Suraci, "Secure mediation gateway architecture enabling the communication among critical infrastructures," in Future Network and Mobile Summit, 2010. IEEE, 2010, pp. 1-8.

[16] P. Capodieci, S. Diblasi, E. Ciancamerla, M. Minichino, C. Foglietta, D. Lefevre, G. Oliva, S. Panzieri, R. Setola, S. De Porcellinis, et al., "Improving resilience of interdependent critical infrastructures via an on-line alerting system," in Complexity in Engineering, 2010. COMPENG'10. IEEE, 2010, pp. 88-90.

[17] — , "Improving resilience of interdependent critical infrastructures via an on-line alerting system," in Complexity in Engineering, 2010. COMPENG'10. IEEE, 2010, pp. 88-90.

[18] M. Lavorato, J. F. Franco, M. J. Rider, and R. Romero, "Imposing radiality constraints in distribution system optimization problems," IEEE Transactions on Power Systems, vol. 27, no. 1, pp. 172-180, 2012.

[19] H. Ahmadi and J. R. Martí, "Mathematical representation of radiality constraint in distribution system reconfiguration problem," International Journal of Electrical Power \& Energy Systems, vol. 64, pp. 293-299, 2015. 